\title{
La metodología didáctica de enseñanza de idiomas en programas para sordos. Un estudio comparado en España y Francia (XVII-Xx)
}

\author{
Antonia Navarro Rincón \\ Universidad de Granada
}

Recibido: 28 febrero 2012 / Aceptado: 24 junio 2012

ISSN: $1697-7467$

\begin{abstract}
RESUMEN: Este artículo expone un estudio comparado en perspectiva metodológico-didáctica, realizado a partir de una investigación sobre programas de enseñanza de idiomas para sordos, publicados en España y Francia, entre los siglos XVII y Xx. Con ese fin, se presenta el marco teórico de referencia del objeto de estudio; se muestran el diseño de la investigación y los resultados; y se explicitan las conclusiones, que ponen de relieve las relaciones entre las metodologías didácticas aplicadas a la enseñanza de idiomas y las utilizadas en los casos seleccionados. Se orienta, además, hacia actuaciones investigadoras en didáctica de la lengua para sordos.
\end{abstract}

Palabras clave: sordos, metodología didáctica, idiomas, programas.

The Didactic Methodology of Language Teaching in the Syllabi for Deaf Students. A Contrastive Study in Spain and France (XVII-XX)

\begin{abstract}
This article presents a comparative study with a methodological-didactic perspective carried out from a piece of research related to languages teaching syllabus addressed to deaf people, and published in Spain and France from the $17^{\text {th }}$ to the $20^{\text {th }}$ century. With this aim, the theoretical reference framework of the object of study is presented, the design of the research and the results are shown, and the conclusions specified show the relationships among the didactic methodologies applied to the languages teaching and those used in selected cases. Moreover, it is oriented towards researching actions in didactics of languages addressed to deaf people.

Keywords: deaf people, didactic methodology, languages teaching, syllabi.
\end{abstract}

\section{INTRODUCCIÓN}

Los estudios sobre la evolución histórica de las metodologías de enseñanza/aprendizaje de idiomas adquieren un valor relevante en el ámbito de la didáctica de las lenguas ${ }^{1}$ (Titone, 1968; Galisson, 1982; Stern, 1983; Besse, 1984 y 1995; Richards y Rodgers, 1998; Christian

\footnotetext{
${ }^{1}$ Los idiomas o lenguas pueden ser concebidos según los usos del término y “(...) la evolución del punto de vista adoptado, de muy diversas maneras (...)” (Suso y Fernández, 2001, 47). Los estudios pueden calificar a los mismos, en función de las variables consideradas, en lengua materna (LM), primera lengua (L1), lengua extranjera (LE), segunda lengua (L2), lengua escolar...
} 
Puren, 1988; Germain, 1993; Coste, 1994; Martínez, 1998; Sánchez, 1998; Fernández y Suso, 1999; Bruña, 2001; Morales, 2003 y Urbano, 2003).

Un aspecto no abordado hasta ahora desde esta disciplina es el relacionado con la evolución de los métodos utilizados en los programas de idiomas para sordos a lo largo de la historia de la educación, a cuya temática dedicaremos los siguientes epígrafes, examinándola en perspectiva diacrónica y comparada, en dos contextos de reconocida trayectoria en el campo nos ocupa: España y Francia.

La revisión de las clasificaciones de los mismos, especialmente las recogidas en los programas que son objeto de estudio, abre el segundo apartado, marco teórico de referencia requerido para contextualizar las cuestiones relacionadas con el diseño de la investigación, que constituye el núcleo del apartado tercero, donde se abordan la metodología seguida, los objetivos e hipótesis, las delimitaciones de contextos y periodos, la muestra, los instrumentos de registro de datos, los resultados y su discusión.

En cuarto lugar, se muestran las conclusiones del estudio comparado de casos y se ofrece una visión sintética de las relaciones establecidas entre la evolución de la metodología didáctica aplicada a la enseñanza de idiomas, en general, y la recogida en los programas seleccionados, a modo de conclusión. Finalmente, se pone de relieve la prospectiva del estudio llevado a cabo.

\section{Métodos de enseñanza de Lenguas en La EduCación de SORdos EN España Y Francia}

Las publicaciones sobre la enseñanza del idioma a sordos surgen con el reconocimiento social de las posibilidades educativas de estas personas. Célebres institutores del siglo XVI, como fray Pedro Ponce de León, y autores coetáneos dejaron constancia por escrito de los logros alcanzados en dicho magisterio, aunque no dieron publicidad a los métodos utilizados, considerados milagrosos y mantenidos en secreto por maestros y discípulos.

En España, la publicación del primer tratado sobre el arte de enseñar a hablar a los "Mvdos" (Pablo Bonet, 1620) rompía con el silencio sobre las prácticas didácticas. Como en iniciativas similares aparecidas en la misma época, en otros países, la obra de Pablo Bonet estuvo sujeta a críticas y acusaciones de plagio del método; bien por ser utilizado anteriormente por otros maestros (Ramírez de Carrión); o por haber sido promovidos en publicaciones pendientes de aprobación (Baptista de Morales, 1623) o en obras ya editadas (Wallis y Amman). Polémicas recogidas en los escritos sobre historia de educación de sordos y que continúan siendo documentadas en la actualidad.

\subsection{El origen de la controversia}

En el Siglo de las Luces, Charles Michel de L’Épée pondrá en marcha la vía de las señas metódicas para facilitar a los sordos la inteligencia de la lengua francesa con la que instruirles en todo tipo de conocimientos e idiomas. La nueva carrera emprendida por el abate de L'Épée, tras el encuentro fortuito con las gemelas sordas, estuvo ligada, desde el comienzo, a la necesidad de defender su exitoso método de las opiniones vertidas en contra 
por maestros de prestigio de la época, como Pereira o Heinicko. Al mismo tiempo, se generó la disputa sostenida con el institutor de Leipzig (Épée, 1784), germen de la controversia oral/gestual, que fue posicionando a defensores y detractores del uso de los signos en la educación de las personas sordas, a lo largo de la historia.

Las publicaciones españolas y francesas, que en el siglo XVIII abordan la temática metodológica (Deschamps, 1779; Épée, 1784; Hervás y Panduro, 1795: Sicard, 1799) esbozan el recorrido histórico del arte de enseñar, destacan la labor realizada por los primeros educadores y debaten sobre las prácticas de predecesores, con la consiguiente toma de posiciones.

El abate Deschamps (1779) distinguirá entre los dos principales sistemas conocidos hasta entonces: el de los signos metódicos del célebre institutor francés y el sistema de la palabra, decantándose por el segundo. Por ello, suscitó la crítica del escritor sordo Pierre Desloges (Desloges, 1779).

\subsection{Instituciones y métodos en el siglo XIX}

La fundación de nuevas instituciones traerá consigo la multiplicación de métodos durante el siglo XIX. Por ello, los trabajos de recopilación y sistematización de los practicados, tanto en Francia como en otros países europeos, se plantearon como necesarios.

El barón Degérando expondrá la forma de clasificarlos en el ensayo que escribió sobre educación (1827), en el que ofrecía las claves para analizarlos, atendiendo a un pequeño número de principios que determinaban sus características. Contemplaba dos partes esenciales o grados en el arte de enseñar a los sordos: por un lado, la elección del instrumento material para remplazar el habla; y, por otro, la enseñanza que daba la inteligencia de la lengua.

En la primera parte, identificaba cinco medios entre los que optar, que, a su vez, agrupaba en dos clases según se ajustasen a las ideas o a las palabras (puesto que pensaba que la enseñanza de la lengua comprendía esos dos elementos y tenía como objetivo asociar el pensamiento a la expresión). Consideraba que el dibujo con su conversión a escritura simbólica y el lenguaje de acción con su conversión a signos metódicos eran los medios que convenían a las ideas. La escritura alfabética, el alfabeto manual o la dactilología y el alfabeto labial acompañado de la pronunciación artificial se adaptaban mejor a las palabras.

En la otra parte del arte de instruir a los sordos, mediante la cual se alcanzaba la inteligencia de la lengua, existían dos maneras de proceder. Bien se seguía, en la medida de lo posible, la misma marcha que en la educación ordinaria, con la división en dos periodos separados y sucesivos: uno, durante el cual quedaba abandonada a las lecciones resultantes del uso práctico, creándose así un primer medio familiar aunque imperfecto de comunicación; y, el otro periodo, que, a continuación y con la ayuda del anterior instrumento, venía a dar una instrucción clásica. O bien, en la segunda manera de proceder, se reunían ambas enseñanzas en una y se introducía al sordo en la inteligencia de la lengua por las vías de la intuición y la lógica. Se instituía de ese modo un método específico para ellos con el que poder comprender la lengua en la que habían aprendido a razonar. Así, los métodos seguidos en la educación de los sordos se clasificaban en dos grandes ramas: unos que se ocupaban esencialmente de la elección y creación del instrumento material; los otros, que dirigían toda la atención hacia la enseñanza filosófica de la lengua (Degérando, 1827).

Más tarde, De Haerne reflexionará también sobre las principales metodologías empleadas en la educación de personas sordas. En su obra (1865), señalaba que los alemanes 
denominaban método francés a aquellos que no tenían la enseñanza de la articulación como elemento esencial, independientemente de las divergencias que existiesen entre ellos. El criterio anterior sirvió para caracterizar a todas las escuelas fundadas en diversas épocas, tanto dentro como fuera de Francia. Pero, como afirmaba este autor, lo que hasta entonces se conocía con tal denominación no debía considerarse un método único y sinónimo del empleado por el abate de L'Épée sino que comprendía una gran variedad de divisiones y subdivisiones dependiendo de los factores considerados, tales como:

1. Los procedimientos pedagógicos (los signos, la escritura y la lectura, la dactilología, los gestos, la articulación y la lectura labial, el dibujo y la intuición de los objetos, hechos y acciones).

2. La importancia relativa que se otorgara a algunos de los anteriores elementos de instrucción.

3. El orden o algoritmo seguido en el empleo de los medios pedagógicos, particularmente los signos y la escritura.

4. El procedimiento dominante o característico de cada uno de los métodos (división generalmente aceptada), distinguiéndose:

- El método basado en los signos naturales utilizados por los propios sordos.

- El que adoptaba los signos metódicos conformes a la naturaleza.

- El que seguía los signos mímicos perfeccionados (es decir, imitando las cosas expresadas).

- El que usaba la pantomima o la imitación hecha con todo el cuerpo.

- El método de la mímica natural (formada por los signos mímicos y los naturales).

- El método intuitivo, que procedía por la intuición o por la simulación de objetos, hechos y acciones o por el lenguaje de acción.

- El de los signos gesticulares que consistía en fundir los signos de los sordos en gestos por los que expresamos nuestros sentimientos reales y en armonizarlos, especialmente desde el punto de vista moral y religioso.

Esta síntesis resulta esclarecedora de la variedad de criterios para la clasificación de los métodos que se venía practicando en Francia.

A lo largo del siglo xIX, en el contexto español, se vino manifestando un creciente interés por los aspectos metodológicos en la enseñanza de las lenguas a los sordos. Así, Tiburcio Hernández (1815) hablaba de la conveniencia de adaptar el método a cada idioma: "Como cada idioma tiene cosas peculiarmente suyas, los métodos han de prescribirse en particular, y las observaciones extrangeras no adoptarse sino despues de haber visto se acomodan al genio del lenguage en que se pretenden ingerir." (Hernández, 1815: III-IV). Y destacaba la conveniencia de seguir para el castellano la doctrina sobre pronunciación de Juan Pablo Bonet, aunque simplificándola previamente. Otros países no se encontraban en el mismo caso y por ello se veían “(...) precisados á preferir el ponerles en estado de comunicación por medio del alfabeto manual y de la escritura. Aquí han de ser aquellos métodos medios supletorios para quando la mudez consista en vicio de los órganos de la voz." (Hernández, 1815: IV).

Ante la cantidad de métodos conocidos, Ballesteros constataba, en la parte teórica de su obra en coautoría (Ballesteros y Fernández, 1845), la imposibilidad de ponerse de acuerdo en considerar a alguno como fijo y universal puesto que, además, cada profesor 
seguía el suyo propio. Tras recomendar el método intuitivo y el sintético para todo género de enseñanza, hacía una revisión de los utilizados principalmente en la educación de sordos, dando una idea sobre ellos y clasificándolos por autores. Así distinguía el método Bonet, el de Pereira, el del abate L'Épée, Sicard y otros como el de Deschamps en Francia; el de Kerger, Raphel y Heinicko en Alemania; el de Wallis en Gran Bretaña y el de Amman en Holanda (Ballesteros y Fernández, 1845).

A finales de siglo, las cuestiones metodológicas en auge, tras el triunfo del oralismo en el Congreso de Milán (1881), eran explicitadas por Faustino Barberá en el capítulo primero de su obra, titulado "Métodos generales de enseñanza.- Medios de comunicación" (Barberá, 1895: 27). Indicaba los siguientes: mímica (natural, signos metódicos, sistema mixto en forma de mímica intermedia entre la natural y la convencional), escritura manuscrita y fugaz, lectura fonética o hablada, visual y táctil; dactilología, palabra (entendida como expresión fonética del pensamiento), lectura labial, dibujo e intuición. En el capítulo siguiente, hacía un análisis crítico de los métodos generales de enseñanza. Distinguía entre los beneficios de la mímica, del método mixto y del método oral, según su división en cuatro grupos de los 'sordos y mudos'. Consideraba que con el primer grupo, los sordos de nacimiento ciegos, solo se podía utilizar el método mixto, formado por la dactilología y la escritura táctil. Con los sordos de nacimiento que veían o con los que oían muy poco, se debía proceder por el método oral puro, es decir, por la intuición y la palabra.

\subsection{Clasificaciones en el siglo $\mathrm{XX}$}

Como ya señalaran Pertusa y Gil (1922), que limitaban a cuatro los métodos o caminos para llegar a la adquisición de conocimientos (analítico, sintético, inductivo y deductivo), ante tanta variedad de clasificaciones pocas podían aceptarse como buenas y apropiadas.

En publicaciones del siglo XX, se planteaban divisiones que aglutinaban métodos y sistemas complementarios, alternativos o aumentativos de comunicación para sordos.

Torres (1988) presentaba de forma esquemática la siguiente agrupación:

1) Verbotonal de Peter Guberina; multisensoriales; audiorales basados en la labiolectura, como La Palabra Complementada; audiorales con representación manual de fonemas, como el método fonomímico de Grosselin y audiorales con dactilología, como el método Rochester.

2) Métodos gestuales, entre ellos: dactilología pura; mímica pura; lenguaje de signos seguido de aprendizaje de la lengua oral y escrita; lenguaje de signos seguido de aprendizaje de la lengua escrita antes que oral.

3) Métodos mixtos, como la comunicación bimodal unilingüe (lengua oral y signos simultáneamente), la comunicación bimodal bilingüe (lengua oral y signada usada alternativamente) y la comunicación total (entendida como una filosofía de la comunicación).

4) Sistemas de apoyo a los métodos orales, a través de sistemas táctiles, visuales o informatizados.

Perelló y Tortosa (1992), en su capítulo noveno dedicado a pedagogía, incluían para la enseñanza de la lengua: la labio-lectura, el lenguaje de signos y los métodos orales (distinguiendo entre los unisensoriales, la estimulación auditiva, los métodos visuales, los procedimientos táctiles y otros procedimientos). 
Teniendo en cuenta el marco teórico presentado, las clasificaciones descritas se alejan de las planteadas en las obras dedicadas a la evolución histórica de la enseñanza de lenguas que se han tomado como referentes en el estudio expuesto en este artículo (Richards y Rodgers, 1998; Puren, 1988; Germain, 1993; Fernández y Suso, 1999 y Urbano, 2003). Las diferencias que se establecen están originadas por los puntos de vista y criterios adoptados así como por las variables consideradas. La investigación realizada desde el ámbito de la didáctica de la lengua, entre otras aportaciones, permitirá el establecimiento de un lenguaje común, en cuanto a metodología didáctica aplicada a la enseñanza de idiomas, que podrá ser compartido por los profesionales vinculados a la educación de personas sordas.

\section{Metodología de LA investigación}

El estudio comparado realizado tiene como punto de partida una investigación que se ha llevado a cabo sobre programas de enseñanza de lenguas para sordos, publicados en España y Francia, entre los siglos xvii y xx. Se ha optado por una metodología cualitativa para abordar las cuestiones que plantea este tipo de investigación.

\subsection{Objetivos e interrogantes}

Los objetivos son:

1) Seleccionar un corpus representativo de programas de los contextos y periodos que abarca este estudio.

2) Analizar cada programa del corpus en su anclaje contextual y temporal, atendiendo a distintas variables que caracterizan el acto didáctico que propician.

3) Inferir la metodología didáctica aplicada a la enseñanza de idiomas de la que participa cada caso.

4) Comparar la evolución de los métodos de enseñanza de idiomas y los seguidos en los programas para sordos seleccionados.

Con fundamento en los objetivos anteriores y dado que la investigación es de carácter exploratorio y cualitativo, las hipótesis, derivadas de los diversos modelos teóricos, equivalen a interrogantes fundamentales sobre los distintos programas y sus características. Los interrogantes se pueden agrupar en los siguientes, de acuerdo con los objetivos de la investigación:

1) ¿Cuáles son los programas representativos de los contextos y periodos delimitados en el estudio, atendiendo a los hitos e influencias más determinantes?

2) ¿Cuál es la caracterización de cada programa según las variables consideradas en el acto didáctico que propician?

3) ¿Cuál es la metodología didáctica aplicada a la enseñanza de idiomas de la que participa cada programa? ¿La evolución metodológica ha sido paralela, en general, a la seguida en España y Francia?

4) ¿Existen diferencias entre los métodos seguidos en los contextos y periodos establecidos? ¿Cuáles son las dimensiones comunes? 


\subsection{Acotaciones y periodizaciones}

Se han considerado los problemas y dificultades que surgen al adoptar una orientación diacrónica y contrastiva. Entre las dificultades se encuentran, en primer lugar, la de definir los límites tanto contextuales o geográficos como temporales; la segunda dificultad nace de la necesidad de la periodización del tiempo acotado; y la tercera, al compatibilizar la periodización en didáctica de la lengua y en historia de la educación de sordos. Se presenta, a continuación, la acotación de estas dimensiones, con la justificación coherente para su estudio.

A. Contexto geográfico: Los contextos de España y Francia son suficientemente representativos. El primero es pionero en el arte de enseñar a hablar a personas sordas, el segundo en educar mediante signos metódicos.

B. Evolución o progresión de los distintos métodos de enseñanza de lenguas. Para ello, se ha considerado el abanico temporal entre los siglos XVI y xx, ambos incluidos. Como fecha de inicio se ha tomado el año de aparición del primer programa español en 1620. El periodo se cierra con el xx, cuando las lenguas de signos comienzan a tener el reconocimiento que les corresponden y se experimentan métodos para su enseñanza.

C. Periodizaciones concretas de la educación de sordos, por un lado, y de evolución de la metodología didáctica de enseñanza de lenguas, por otro; así como de aquellas que permitan compatibilizarlas.

En primer lugar, se han contemplado las periodizaciones en obras sobre historia de educación de sordos:

a) La división de García Pico de Ponce (1981).

b) La periodización que realiza González Moll (1992).

c) La que se recoge en Moody y otros (1998).

En segundo lugar, para determinar los periodos en la evolución de la enseñanza de idiomas hemos acudido a estas propuestas:

a) Puren (1988).

b) Germain (1993).

c) Richards y Rodgers (1998).

d) Fernández y Suso (1999).

Para integrar las periodizaciones de ambos ámbitos, por razones de conveniencia para el estudio que aquí se expone, se ha optado finalmente por la adoptada por Germain, adaptada por Urbano (2003). De acuerdo con la misma, se determinan tres periodos cronológicos. Como no aparecen programas publicados para enseñar idiomas a sordos en el primero, no se ha considerado. Solo se han tenido en cuenta, pues, los dos siguientes: el que comprende los siglos XVI-XIX y el que abarca el XX.

\subsection{Muestra}

Se ha seleccionado, en total, una veintena de programas de los contextos y periodos delimitados. El "muestreo" o elección de los objetos de investigación ha consistido en una 
selección intencionada de los casos de estudio. De hecho, suele calificarse como "teórico, motivado, no probabilístico accidental o intencional" (Tojar, 2006: 186). Esta técnica de muestreo no arbitrario ni estadístico es la principal estrategia de selección en el estudio de casos. Cohen y Manion (1990) la denominan muestra de conveniencia. La elección se produjo tras haber contemplado la fase de construcción de un cuerpo teórico sobre el contenido a investigar que permitió sacar a la luz un repertorio de programas publicados. Se han excluido de la muestra los programas específicos para personas que disponen de restos auditivos; también se han descartado aquellos que no son originarios del contexto francés o español, es decir, las traducciones o adaptaciones de métodos creados en otros países ${ }^{2}$.

En cuanto a la distribución, se han considerado trece programas correspondientes al periodo que comprende los siglos XVI-XIX, ambos incluidos, lo que constituye el $65 \%$ del total de programas analizados, versus el $35 \%$ de los siete programas correspondientes al último periodo determinado para el siglo XX.

Según los contextos y la evolución por siglos, se han seleccionado dos programas del primer periodo, correspondiente al XVII, el 10\% del total de los analizados. Ambos son españoles porque en Francia no aparecen las primeras publicaciones hasta un siglo más tarde.

Otros cuatro programas corresponden al siglo XVIII, esto es, el $20 \%$ del total de la muestra. Arranca, con fuerza, en el país galo la dedicación a la enseñanza de sordos y con ella la producción de obras. Por ello, y con propósito de compensar la carencia en el siglo anterior, tres programas son seleccionados del contexto francés y uno, bien representativo de la época, del español.

En el siglo XIX proliferan los programas de relevancia y representatividad en ambos contextos. Con ese fundamento, se han analizado siete programas, es decir, el 35\% del total. De ellos, cuatro pertenecen al panorama francés y tres al español.

Sin duda, el mayor número de este tipo de publicaciones corresponde al siglo XX; no solo en frecuencia sino también en variedad. De este periodo se han extraído siete programas: el $35 \%$ del total, siendo cuatro españoles y el resto del contexto francés.

El periodo primero (XVI-XIX) es notablemente más amplio, de ahí que el porcentaje también lo sea, sin embargo, el periodo último (XX) es más variado.

\subsection{Instrumentos}

Con el propósito de sistematizar la información resultante de la búsqueda documental realizada, se ha empleado la ficha de registro que presentamos en el cuadro 1. Esta ha sido completada con los datos de cada uno de los programas seleccionados.

\footnotetext{
${ }^{2}$ La muestra definitiva o corpus representativo se encuentra contenido en estas obras: Pablo Bonet, 1620; Baptista de Morales, 1623; Deschamps, 1776; Épée, 1784; Sicard, 1799; Hervás y Panduro, 1795; Hernández, 1815; Bébian, 1827; Ballesteros y Fernández, 1845; Valade-Gabel y Valade-Gabel, 1879; Grosselin, 1881; Barberá, 1895; Thollon, 1898; Lauer, 1920; Granell y Forcadell, 1928; Perelló y Tortosa, 1992 [1968]; Moody y otros, 1998 [1983]; Perelló y Frigola, 1998 [1986]; Alonso y otros, 1989; Istria y otros, 1992.
} 
Cuadro 1. Ficha de registro

\begin{tabular}{|l|l|}
\hline Título del programa & \\
\hline Año / Siglo & \\
\hline Autor/Breve biografía y obras del autor & \\
\hline Localización (editorial y lugar de edición) & \\
\hline Relevancia histórica & \\
\hline Contexto en el que se diseñó & \\
\hline Contexto en el que se implementó & \\
\hline Finalidad principal & \\
\hline Fuentes & \\
\hline Lengua & \\
\hline Estructura & \\
\hline Metodología & \\
\hline Actividades & \\
\hline Recursos & \\
\hline Alumnado & \\
\hline Evaluación & \\
\hline Potencialidad & \\
\hline Defectos & \\
\hline Innovación & \\
\hline Resultados del programa & \\
\hline Profesionales que lo imparten & \\
\hline Objetivos & \\
\hline Contenidos básicos & \\
\hline Nivel/es & \\
\hline Otros... (Indicar cuáles) & \\
\hline
\end{tabular}

\subsection{Resultados y discusión}

Una vez recopilada la información necesaria se llevó a cabo el tratamiento de la misma mediante el procedimiento de análisis del contenido, centrado en cada uno de los programas, en un primer momento; y más tarde, realizándolo entre los casos, de forma colectiva.

Teniendo en cuenta la periodización finalmente establecida y situados en el primer periodo de este estudio se observan semejanzas y diferencias en las metodologías de las que participan los casos seleccionados. Se aprecia igualmente la idiosincrasia de los programas para sordos, en cuanto a la utilización de medios y recursos específicos, según los contextos y épocas.

\subsubsection{Los programas españoles del siglo XVII}

Pablo Bonet (1620) comienza a emplear como recurso didáctico las imágenes o grabados del alfabeto manual, encontrando en lo visual el camino llano con el que suplir la carencia de oído en la enseñanza de idiomas, no solo del castellano sino también del griego. Aplica 
el método tradicional práctico de enseñanza de idiomas. El programa de Baptista de Morales (1623) sigue un método tradicional teórico, presentando las reglas gramaticales de forma explícita y los ejercicios memorísticos y repetitivos para el aprendizaje de la lengua. Ambos programas para sordos comparten la metodología tradicional de enseñanza de lenguas, según se desprende al cotejar los datos obtenidos con la descripción de la caracterización que de los mismos realizan distintos autores (Germain, 1993; Fernández y Suso, 1999).

La particularidad de los empleados por los preceptores de sordos, (que no difieren en esencia de los usados con oyentes en este siglo) consiste en la utilización del alfabeto manual para el deletreo y en la reducción de la nominación de las letras a la pronunciación de sus elementos primitivos para abordar al mismo tiempo la enseñanza de la lectoescritura y la pronunciación. Sin duda, en ello estriba la originalidad y el acierto de los primeros programas. XVIII

3.5.2. La metodología tradicional de enseñanza de lenguas en los programas del

En el contexto francés, el programa de Deschamps (1776), se enmarca en el preceptorado. Aplica el método tradicional teórico de enseñanza de lenguas. Su finalidad es propiciar la pronunciación y la comunicación oral en las personas sordas a través del aprendizaje de la lectura labial. La particularidad del método estriba en la utilización del alfabeto manual y del tacto en la comunicación de sordos en la oscuridad, por lo que también resultará funcional para personas sordociegas.

Los siguientes casos se enmarcan en la misma línea metodológica tradicional de enseñanza de lenguas y presentan las variantes propias, según las clasificaciones establecidas por los especialistas en la materia.

El programa del abate de L’Épée (1784) propicia los primeros aprendizajes con el método tradicional de gramática traducción aunque algunos procedimientos son propios del método tradicional práctico de enseñanza de lenguas. De L’Épée, propone como finalidad prioritaria enseñar a pensar de forma estructurada en la lengua francesa con la que adquirir nuevos conocimientos e idiomas. La peculiaridad del método reside en usar el lenguaje de acción, natural entre sordos, y en crear a partir de él los signos metódicos como puente entre lenguas y metalenguaje en la enseñanza del oral y el escrito. Ésta es, sin duda, su mayor aportación y contribución a la comunicación de personas sordas. En este sentido, se le ha considerado precursor de la modalidad de comunicación conocida en los años ochenta del pasado siglo como idioma signado o comunicación bimodal.

El programa elaborado por el español Lorenzo Hervás y Panduro (1795) continúa la tendencia del abate de L'Épée, es decir, se aprecia una metodología tradicional aunque en la variante de su método, considerado práctico, dentro del marco general de la metodología tradicional. La peculiaridad consiste en el orden seguido en enseñanza de las destrezas. Los sordos son iniciados en el español escrito. El oral se aborda posteriormente.

\subsubsection{De la metodología tradicional a la directa}

En el contexto español, el plan para sordos de Tiburcio Hernández (1815) no se aparta de la metodología teórica tradicional de enseñanza de lenguas. El programa de Ballesteros 
y Fernández (1845) se institucionalizará en el Colegio de Madrid, participando del método tradicional práctico-teórico.

En el contexto francés también permanece la apuesta por la metodología tradicional en sus distintas variantes, de manos de los autores Sicard y Bébian. El programa de Sicard (1799) tiene como punto de partida la utilizada por L'Épée, aunque realizará sobre su base modificaciones notables. Relata la experimentación de su método con el sordo, Massieu, aconsejando generalizar su uso también con oyentes, como indica en el subtítulo de la obra que lo contiene. En realidad se trata de un curso de metafísica y gramática experimentales propias para la instrucción de todos los niños. Bébian (1827) elabora un programa de corte tradicional, que continúa en parte la tendencia de Sicard, de quien fue discípulo. Se trata de aprender de forma práctica, concretamente por el método práctico-teórico de enseñanza de idiomas. Además, reconoce el lenguaje natural de los sordos y lo emplea para demandar traducciones de una a otra lengua, evaluando de esta manera la comprensión de la lengua francesa.

Con el programa de Valade-Gabel y Valade-Gabel (1879) se promueve en Francia, la corriente educativa de sordos fundamentada en la intuición y en la metodología directa (con los métodos natural, intuitivo y ocasional), cuya tendencia continuará en los programas analizados. El de Grosselin (1881) prima el carácter imitativo de las expresiones para propiciar el adecuado modelado del lenguaje oral. Se enfatiza la repetición de gestos y de acciones en las actividades para afianzar los conocimientos adquiridos y los avances logrados. En todo el proceso, el alumno desempeña un papel activo. El programa de Thollon (Thollon, 1898) cierra en Francia el siglo XIX y el primer periodo considerado en nuestro estudio. En él se opta por un método oral, intuitivo, ocasional y maternal, es decir, utiliza situaciones y estrategias comunicativas similares a las que usan las madres en los intercambios comunicativos con sus hijos. En la portada de su obra inserta una cita del método natural intuitivo u objetivo puesto en práctica por Pestalozzi y su discípulo el Padre Girard.

En el programa diseñado por el valenciano Barberá (1895) se hace explícita la intención del autor de enmarcarlo en la metodología oral pura. De hecho, recoge los principios consolidados en el congreso de Milán, suprimiendo el uso de signos en la educación de sordos y mostrándose partidario de la metodología oralista profesada por el italiano Julio Tarra. Las actividades propuestas confirman que se trata de un método directo e intuitivo. El congreso de 1880 coincide con el establecimiento de la metodología directa de enseñanza de idiomas, el auge de los estudios de fonética y la creación del Alfabeto Fonético Internacional, entre otros acontecimientos relevantes para la educación lingüística en general, y de sordos, en particular.

\subsubsection{Métodos y enfoques de enseñanza de lenguas en los programas del XX}

Se aprecia la continuidad en la apuesta por el método oral y acústico en el único programa de una mujer, M. Lauer (1920), que abre el periodo que comprende el siglo xx. La autora francesa, que es sorda, pretende reeducar la audición de las personas que la han perdido tras la adquisición del lenguaje, es decir, este programa está dirigido en particular a los sordos postlocutivos.

El programa de Granell y Forcadell (1928) se basa en el método directo, en el método maternal de Girad y en el de Mayo, discípulos de Pestalozzi. Se define como intuitivo, oralista y ocasional y lo denomina "método teórico-práctico de enseñanza de idiomas" (Ibid.). 
Es un método ecléctico. Todas las lecciones tienen una estructura idéntica y característica del método de gramática traducción. Utiliza el método oral y el método activo, solicitando que el alumno realice las acciones expresadas por el verbo en forma imperativa.

Alonso y otros (1989) diseñan su programa para los niños sordos educados en centros de integración y sigue un enfoque comunicativo, con intención de que se establezca una comunicación real, aprovechando todos los recursos de las personas que padecen una deficiencia auditiva: la comunicación total.

Los programas elaborados por Perelló en coautoría engloban distintas metodologías. Por un lado, el de Perelló y Tortosa (1992) se enmarca dentro de lo que sería el método oral, aunque desde una perspectiva muy peculiar, dado que estructura sus enseñanzas en el sistema educativo del momento, en concreto, en la etapa de la Educación General Básica.

Por otra parte, el programa presentado por Perelló y Frigola (1998), Lenguaje de signos manuales, toma como punto de partida la enseñanza de la lengua de signos. En el enfoque adoptado, estas se consideran propias de las comunidades sordas y, desde un enfoque educativo bilingüe, las personas sordas se alfabetizan en la lengua oral escolar. En cuanto a la metodología didáctica de enseñanza de las lenguas, en este programa se emplea un método pedagógico basado en la lectura de textos codificados para poder enseñarla en su vertiente escrita a través de la lengua de signos o viceversa. Pretende servir además para que los oyentes aprendan a comunicarse en ella, respetando la estructura de cada lengua.

El manual de lectura labial de Istria y otros (1992) adapta a la lectura labial los elementos audiovisuales de enseñanzas de lenguas extranjeras. Es un método para sordos postlocutivos que dominan la lengua de la comunidad oyente. No sigue el enfoque analítico tradicional que considera cada elemento, ya sea consonante o vocal, a través de sus asociaciones. Parte del principio siguiente: elementos que han sido considerados tradicionalmente como visibles no lo son de forma estable, sino que dependen de sus asociaciones. De ahí que en el programa se incluyan ejercicios que sirven de ayuda para interpretar y completar mentalmente el mensaje. En este método, la vista es fundamental en la discriminación de sonidos de estímulo visual idéntico o similar. También se enfatiza el trabajo de interpretación de significados según el contexto lingüístico y la situación extralingüística.

Por último, el programa publicado por Moody y otros (1998) propone contribuir al desarrollo de la identidad cultural de la comunidad sorda y consolidar el aprendizaje de la Lengua de Signos Francesa (LSF) dentro del marco de la educación bilingüe. Pone en marcha actividades lúdicas como la representación teatral. Se restringe el uso de la lengua oral (regla del silencio) para emplear exclusivamente la de signos y, en todo caso, la expresión de gestos, posturas, etc. Paulatinamente va introduciendo la enseñanza gramatical por medio de traducciones en ambas lenguas. Metodológicamente es destacable la inmersión de los aprendices en la lengua que se pretende enseñar. Coincide con el anterior programa en la tendencia contemporánea de implicar en el aprendizaje de la LSF, a padres de niños sordos $\mathrm{y}$ otros adultos y profesionales interesados.

\section{Conclusiones y prospectiva del estudio}

El estudio comparado que se ha realizado a partir de la investigación que acabamos de presentar permite afirmar que los métodos de los programas para sordos seleccionados, en sendos contextos, siguen la caracterización y progresión documentada en los estudios 
sobre evolución de la metodología didáctica aplicada a la enseñanza de idiomas. El cuadro 2 muestra las conclusiones, de forma sintética.

Cuadro 2. Metodología didáctica en los programas seleccionados

\begin{tabular}{|c|c|}
\hline PROGRAMA & MÉTODO \\
\hline $\begin{array}{l}\text { Redvction de las letras, y arte para enseñar a } \\
\text { ablar los mvdos } \\
\qquad \text { (Pablo Bonet, 1620) }\end{array}$ & Método tradicional práctico \\
\hline $\begin{array}{l}\text { Pronvnciaciones generales de lengvas } \\
\qquad \text { (Baptista de Morales, 1623) }\end{array}$ & Método tradicional teórico \\
\hline $\begin{array}{l}\text { Cours élémentaire d'education des sourds et } \\
\text { muets } \\
\text { (Deschamps, 1776) }\end{array}$ & Método tradicional teórico \\
\hline $\begin{array}{l}\text { La véritable maniere d'instruire les Sourds et } \\
\text { Muets } \\
\qquad \text { (Épée, 1784) }\end{array}$ & Método tradicional teórico-práctico \\
\hline $\begin{array}{l}\text { Escuela española de Sordomudos ó Arte de en- } \\
\text { señarles á escribir y hablar el idioma español } \\
\text { (Hervás y Panduro, 1795) }\end{array}$ & Método tradicional práctico \\
\hline $\begin{array}{l}\text { Cours d'instruction d'un sourd-muet de nais- } \\
\text { sance } \\
\text { (Sicard, 1799) }\end{array}$ & Método tradicional teórico-práctico \\
\hline $\begin{array}{l}\text { Plan de enseñar a los sordomudos el idioma } \\
\text { español" } \\
\qquad \text { (Hernández, 1815) }\end{array}$ & Método tradicional teórico \\
\hline $\begin{array}{l}\text { Manuel d'enseignement pratique des sourds- } \\
\text { muets } \\
\qquad \text { (Bébian, 1827) }\end{array}$ & Método práctico-teórico \\
\hline $\begin{array}{r}\text { Curso elemental de instrucción de sordo-mudos } \\
\text { (Ballesteros y Fernández, 1845) }\end{array}$ & Método práctico-teórico \\
\hline $\begin{array}{l}\text { Plan d'études. Programme de l'enseignement } \\
\text { pour les écoles de sourds-muets non subven- } \\
\text { tionnées par l'état" } \\
\text { (Valade-Gabel y Valade-Gabel, 1879) }\end{array}$ & $\begin{array}{l}\text { Metodología directa } \\
\text { (método intuitivo) }\end{array}$ \\
\hline Manuel de la phonomimie $\quad$ (Grosselin, 1881) & $\begin{array}{c}\text { Metodología directa } \\
\text { (métodos activo, imitativo, repetitivo) }\end{array}$ \\
\hline $\begin{array}{l}\text { La enseñanza del sordomudo según el método } \\
\text { oral } \\
\text { (Barberá, 1895) }\end{array}$ & $\begin{array}{c}\text { Metodología directa } \\
\text { (método oral, método intuitivo) }\end{array}$ \\
\hline
\end{tabular}




\begin{tabular}{|c|c|}
\hline $\begin{array}{l}\text { Premières leçons de choses et de langue } \\
\text { française } \\
\text { (Thollon, 1898) }\end{array}$ & $\begin{array}{l}\text { Metodología directa } \\
\text { (método intuitivo) }\end{array}$ \\
\hline $\begin{array}{l}\text { Conseils aux sourds par un sourd, manuel de } \\
\text { rééducation auditive par la parole et les sons } \\
\text { musicaux } \\
\text { (Lauer, 1920) }\end{array}$ & Método oral \\
\hline $\begin{array}{l}\text { Método teórico-práctico de idioma para la } \\
\text { enseñanza del mismo al sordomudo, por medio } \\
\text { de la palabra hablada } \\
\qquad \text { (Granell y Forcadell, 1928) }\end{array}$ & Método ecléctico \\
\hline $\begin{array}{l}\text { Sordera profunda bilateral prelocutiva } \\
\text { (Perelló y Tortosa, } 1992 \text { [1968]) }\end{array}$ & Método oral \\
\hline $\begin{array}{l}\text { La langue des signes } \\
\qquad \text { (Moody y otros, } 1998 \text { [1983]) }\end{array}$ & $\begin{array}{l}\text { Educación bilingüe (lengua de signos francesa/ } \\
\text { francés escrito) método silencioso }\end{array}$ \\
\hline $\begin{array}{l}\text { Lenguaje de signos manuales } \\
\qquad \text { (Perelló y Frigola, } 1998 \text { [1987]) }\end{array}$ & $\begin{array}{c}\text { Educación bilingüe } \\
\text { (lengua de signos/lengua escrita) }\end{array}$ \\
\hline $\begin{array}{l}\text { Introducción a la Comunicación Bimodal } \\
\qquad \text { (Alonso y otros, 1989) }\end{array}$ & $\begin{array}{l}\text { Enfoques comunicativos } \\
\text { (comunicación total) }\end{array}$ \\
\hline $\begin{array}{l}\text { Manuel de lecture labiale } \\
\text { (Istria y otros, 1992) }\end{array}$ & Método estructuro-global audio-visual \\
\hline
\end{tabular}

La perspectiva adoptada establece un marco de referencia para futuras investigaciones y colaboraciones entre especialistas en didáctica de las lenguas y profesionales vinculados a la educación de sordos, no solo en estudios sobre España y Francia, sino también en los que pudieran llevarse a cabo en otros contextos y periodos.

\section{REFERENCIAS BIBLIOGRÁFICAS}

Alonso, P. et al. (1989). Introducción a la comunicación bimodal. Madrid: MEC, CNREE.

Ballesteros, J. M. y Fernández Villabrille, F. (1845). Curso elemental de instrucción de sordomudos. Madrid: Imprenta del Colegio de Sordo-mudos y Ciegos.

Baptista de Morales, J. (1623). Pronvnciaciones generales de Lengvas, Ortografia, Efcvela de Leer, Efcriuir, y Contar, y fignificación de Letras en la Mano. Montilla: Calle de la Imprenta.

Barberá, F. (1895). La enseñanza del sordomudo según el método oral. Valencia: Imprenta de Manuel Alufre.

Bébian, R. A. A. (1827). Manuel d'enseignement pratique des sourds-muets. Paris: Méquignon l'Aîné, Père.

Besse, H. (1984). Grammaire et didactique des langues. Paris: Hatier-Crédif. 
(1995). Méthodes et pratiques des manuels de langue. Paris: Didier-Crédif.

Bruña Cuevas, M. (2001). "L'enseignement du français mis au service du régime de Franco (19361940). Changements politiques et épistémologie 1780-1945", en Rodopi, 303-23.

Cohen, L. y Manion, L. (1990). Métodos de investigación educativa. Madrid: La Muralla.

Coste, D. (1994). Vingt ans dans l'évolution de la didactique des langues. Paris: Crédif-Hatier.

Degérando, M. (1827). De l'éducation des sourds-muets de naissance. Paris: Chez Méquignon l'ainé père, éditeur.

Deschamps, A. (1779). Cours élémentaire d'éducation des sourds et muets, suivi d'une dissertation sur la parole, traduite du latin de Jean-Conrad Amman, par M. Beauvais de Préau. Paris: Chez les frères Debure.

Épée, CH. M. de L' (1784). La véritable manière d'instruire les sourds et muets, confirmée par une longue expérience. Paris: Chez Nyon.

Fernández Fraile, M. E. y Suso López, J. (1999): La enseñanza del Francés en España (17671936). Granada: Método Ediciones.

Galisson, R. (1980). D'hier à aujourd'hui. La didactique des langues étrangères. Du structuralisme au fonctionnalisme. Paris: CLE International.

Galisson, R. (1982). D'autres voies pour la didactique des langues étrangères. Paris: HatierCrédif.

García Pico de Ponce, O. (1981). Historia de la educación de sordos. Buenos Aires: Publicaciones Médicas Argentinas.

Germain, C. (1993). Évolution de l'enseignement des langues: 5000 ans d'histoire. Paris: CLE International.

González Moll, G. (1992). Historia de la educación del sordo en España. Valencia: Nau llibres.

Granell y Forcadell, M. (1928). Método teórico-práctico de idioma para la enseñanza del mismo al sordomudo, por medio de la palabra hablada. Madrid: Imprenta del Colegio Nacional de Sordomudos y de Ciegos.

Grosselin, A. (1905) [1881]. Manuel de la phonomimie ou méthode d'enseignement par la voix et par le geste. Paris: Belin Frères, $7^{\mathrm{e}}$ édition; $1^{\mathrm{e}}$ édition, 1881.

Haerne, De (1863). De l'enseignement spécial des sourds-Muets, considéré, dans les méthodes principales, d'après la tradition et le progrès. Bruxelles: Comptoir Universal d'Imprimerie et Librairie Victor Devaux et Cie.

Hernández, T. (1815). Plan de enseñar a los sordo-mudos el idioma español. Madrid: Imprenta Real.

Hervás y Panduro, L. (1795). Escuela española de sordomudos ó arte para enseñarles á escribir y hablar el idioma español. T. I, Madrid: Imprenta Real. T. II, Madrid: Imprenta de Fermín Villalpando.

Istria, M. y otros (1992). Manuel de lecture labiale. Exercices d'entraînement. Paris: Masson.

Lauer, M. (1920). Conseils aux sourds par un sourd, manuel de rééducation auditive par la parole et les sons musicaux. Paris: A. Maloine et fils.

Martínez, P. (1998). La didactique des langues étrangères. Paris: PUF.

Moody, B. y otros (1998) [1983]. La langue des signes. Vincennes: IVT.

Morales Gil, F. J. (2003). La evolución metodológica de la enseñanza oficial del francés en España: 1936-1970. Tesis doctoral. Huelva: Universidad de Huelva.

Navarro Rincón, A. (2011). La enseñanza de idiomas a sordos. Estudio de programas en España y Francia $(X V I I-X X)$. Tesis doctoral. Universidad de Granada. 
Pablo Bonet, J. (1620). Redvction de las letras y arte para enseñar a ablar los mvdos. Madrid: Francisco Abarca de Angvlo.

Perelló, J. y Frigola, J. (1998) [1987]. Lenguaje de signos manuales, Madrid: CIE Inversiones Editoriales Dossat 2000.

Perelló, J. y Tortosa, F. (1992) [1968]. Sordera profunda bilateral prelocutiva. Barcelona: Masson, 4. ${ }^{\mathrm{a}}$ ed.

Pertusa, V. y Gil, A. (1922). Pedagogía Moderna. T. II: Tratado de la Educación. T. III: Historia de la Educación y de la Pedagogía. Málaga: Tip. y Lit. de R. Alcalá.

Puren, CH. (1988). Histoire des méthodologies de l'enseignement de langues. Paris: CLE International.

Richards, J. C. y Rodgers, T. S. (1998) [1986]. Enfoques y métodos en la enseñanza de idiomas. Madrid: Cambridge University Press.

Sánchez Pérez, A. (1998). Los métodos en la enseñanza de los idiomas. Madrid: SGEL.

Sicard, R. A. (1799). Cours d'instruction d'un sourd-muet de naissance, et qui peut être utile à l'Éducation de ceux qui entendent et qui parlent avec figures et tableaux. Paris: Chez Le Clere.

Stern, H. H. (1983). Fundamental Concepts of Language Teaching. Oxford: Oxford University Press.

Thollon, B. (1898). Le deuxième livre du sourd-parlant. Premières leçons de choses et de langue française. Paris: Georges Carré et C. Naud.

Titone, R. (1968). Teaching Foreign Languages: An Historical Sketch.Washington, D. C.: Georgetown University Press.

Tojar, J. (2006). Investigación cualitativa: comprender y actuar. Madrid: La Muralla.

Torres Monreal, S. (1988). La Palabra complementada. Madrid: CEPE.

Urbano Marchi, B. (2003). La enseñanza-aprendizaje del francés como lengua extranjera en España durante la época franquista (1938-1970). Tesis doctoral. Universidad de Granada.

Valade-Gabel, J. J. y Valade-Gabel A. (1879). Plan d'études. Programme de l'enseignement pour les écoles de sourds-muets non subventionnées par l'état. Paris: Delagrave. 